Homology, Homotopy and Applications, vol.14(1), 2012, pp.281-285

\title{
THE ISOMORPHISM BETWEEN MOTIVIC COHOMOLOGY AND $K$-GROUPS FOR EQUI-CHARACTERISTIC REGULAR LOCAL RINGS
}

\author{
YUKI KATO \\ (communicated by J.F. Jardine)
}

\begin{abstract}
One of the well-known problems in algebraic $K$-theory is the comparison of higher Chow groups and $K$-groups. In this paper, using the motivic complex defined by VoevodskySuslin-Friedlander, we prove the comparison theorem for equicharacteristic regular local rings.
\end{abstract}

\section{Introduction}

Voevodsky-Suslin-Friedlander [8] defined the motivic cohomology $\mathrm{CH}_{\mathrm{Zar}}^{r}(X, n)$ by using equi-dimensional cycle groups $\mathcal{Z}_{\text {equi }}\left(X \times \Delta^{\bullet} \times \mathbb{A}^{r} / X \times \Delta^{\bullet}, 0\right)$ for smooth noetherian schemes $X$ over a field and showed the contravariant functoriality for morphisms of schemes. Friedlander-Suslin [2] proved that $\mathrm{CH}_{\text {Zar }}^{r}(X, n)=\mathrm{CH}^{r}(X, n)$ for smooth quasi-projective schemes $X$ over a field, where $\mathrm{CH}^{r}(X, n)$ is the higher Chow group of $X$ defined by Bloch [1]. For smooth quasi-projective schemes $X$ over a field, Bloch [1] proved that $\bigoplus_{r \geqslant 0} \mathrm{CH}^{r}(X, n)$ coincides with the $n$-th algebraic $K$-group $K_{n}(X)$ after tensoring with $\mathbb{Q}$. We use the subscript $-\mathbb{Q}$ to mean $-\otimes_{\mathbb{Z}} \mathbb{Q}$.

In this paper, we consider the motivic cohomology groups $\mathrm{CH}_{\mathrm{Zar}}^{r}(X, n)$ for regular schemes by using an equi-dimensional cycle group [8] and prove that there is an isomorphism between the $K$-group $K_{n}(X)$ and the motivic cohomology group $\mathrm{CH}_{\text {Zar }}^{r}(X, n)$ for the spectrum of an arbitrary regular local ring containing a field after tensoring with $\mathbb{Q}$.

Theorem 1.1. Let $R$ be a regular local ring containing a prime field. Then the cycle class map

$$
\mathrm{cl}^{(r)}: K_{n}(R)_{\mathbb{Q}}^{(r)} \rightarrow \mathrm{CH}_{\mathrm{Zar}}^{r}(R, n)_{\mathbb{Q}}
$$

is an isomorphism for any $n, r \geqslant 0$, where $\mathrm{cl}^{(r)}$ is the cycle-class map constructed in Section 3.1 and $K_{n}(R)_{\mathbb{Q}}^{(r)}$ is the eigenspace of the Adams operation $\Psi^{k}: K_{n}(R)_{\mathbb{Q}} \rightarrow$ $K_{n}(R)_{\mathbb{Q}}$ with the eigenvalue $k^{r}$ for $k=2,3, \ldots$

This theorem is proved by using Popescu's result [6, Corollary 2.7] that says that any equi-characteristic regular local ring $R$ is a directed inductive limit of smooth

Received January 25, 2011, revised July 21, 2011; published on June 8, 2012. 2000 Mathematics Subject Classification: 19E15, 14F42, 14C25.

Key words and phrases: algebraic cycles, higher Chow groups, algebraic $K$-theory.

Article available at http://intlpress.com/HHA/v14/n1/a14 and doi:10.4310/HHA.2012.v14.n1.a14

Copyright (C) 2012, International Press. Permission to copy for private use granted. 
sub-algebras $R_{\alpha}$ over a field $F$. Since we may assume that $F$ is perfect $R=\lim R_{\alpha}$ and $K_{n}(R)=\lim K_{n}\left(R_{\alpha}\right)$, we can reduce Theorem 1.1 to the case of a smooth $F$-algebra $R$. Then we have to prove that the functor $\mathrm{CH}_{\mathrm{Zar}}^{r}(-, n)_{\mathbb{Q}}$ commutes with directed inductive limits of algebras, and this is proved by Proposition 2.2.

\section{Acknowledgements}

I would like to express thanks to Professor Masaki Hanamura for his valuable advice. I wish to deeply thank the anonymous referee for very valuable suggestions and comments.

\section{Motivic cohomology of equi-dimensional cycles}

In this section, we always assume that all schemes are regular noetherian and separated. A morphism $p: X \rightarrow S$ of schemes of finite type is said to be equi-dimensional of dimension $r$, if $\operatorname{dim} p^{-1}(p(x))=r$ for any $x \in X$ and any irreducible component of $X$ dominates an irreducible component of $S$. In particular, any equi-dimensional morphism of dimension zero is a quasi-finite morphism and dominates an irreducible component.

Let $\mathcal{Z}_{\text {equi }}(X / S, r)$ be the free abelian group generated by closed integral subschemes of $X$ which are equi-dimensional of dimension $r$ over $S$. We call $\mathcal{Z}_{\text {equi }}(X / S, r)$ the equi-dimensional cycle group of dimension $r$.

Let $X$ be an $S$-scheme of finite type. According to [8, Chapter 2, Theorem 3.3.1, Lemma 3.3.6 and Corollary 3.4.3], for any morphism of regular noetherian schemes $f: T \rightarrow S$, we have a homomorphism $f^{*}: \mathcal{Z}_{\text {equi }}(X / S, r) \rightarrow \mathcal{Z}_{\text {equi }}\left(X \times{ }_{S} T / T, r\right)$ and $\mathcal{Z}_{\text {equi }}\left(X \times_{S}-/-, r\right)$ is a contravariant functor for morphisms of regular noetherian schemes. Furthermore, the functor $\mathcal{Z}_{\text {equi }}\left(X \times_{S}-/-, r\right)$ is an étale-sheaf $[2$, p. 816] on $S$, hence this is a Zariski-sheaf on $S$. We define the motivic cohomology $\mathrm{CH}_{\text {Zar }}^{r}(X, n)$ for finite dimensional regular noetherian schemes $X$ :

Definition 2.1. Let $X$ be a regular noetherian scheme of finite dimension. Write $\Delta^{n}=\operatorname{Spec} \mathbb{Z}\left[t_{0}, \ldots, t_{n}\right] /\left(t_{0}+\cdots+t_{n}-1\right)$. Then $X \times \Delta^{\bullet}$ is a regular, noetherian cosimplicial scheme in the usual sense, and $\mathcal{Z}_{\text {equi }}\left(-\times \Delta^{\bullet} \times \mathbb{A}^{r} /-\times \Delta^{\bullet}, 0\right)$ is a simplicial sheaf on $X$. We define the motivic cohomology to be the Zariski-hypercohomology:

$$
\mathrm{CH}_{\text {Zar }}^{r}(X, n)=\mathbb{H}_{\text {Zar }}^{-n}\left(X, \mathcal{Z}_{\text {equi }}\left(-\times \Delta^{\bullet} \times \mathbb{A}^{r} /-\times \Delta^{\bullet}, 0\right)\right) \text {. }
$$

Let $\left(T_{\alpha}, f_{\alpha \beta}\right)$ be a directed inverse system of smooth schemes over a regular noetherian scheme $S$ with a directed ordered index set $I$, where each transition map $f_{\alpha \beta}: T_{\beta} \rightarrow T_{\alpha}$ is affine and dominant $(\beta \geqslant \alpha)$. Assume that $T=\lim _{\longleftarrow} T_{\alpha}$ is regular and noetherian. Then we have the following:

Proposition 2.2. Let $X$ be a scheme of finite type over $T$ and assume that there exists a scheme $X_{0}$ of finite type over $S$ such that $X=X_{0} \times_{S} T$. Then the canonical morphism of Zariski sheaves on $T$

$$
\underset{\alpha}{\lim }\left(f_{\alpha}^{*} \mathcal{Z}_{\text {equi }}\left(X_{\alpha} \times_{T_{\alpha}}-/-, 0\right)_{\mathbb{Q}}\right) \rightarrow \mathcal{Z}_{\text {equi }}\left(X \times_{T}-/-, 0\right)_{\mathbb{Q}}
$$

is an isomorphism, where $f_{\alpha}: X \rightarrow X_{\alpha}=X_{0} \times_{S} T_{\alpha}$ denotes the canonical morphism 
induced by $T \rightarrow T_{\alpha}$ and $f_{\alpha}^{*} \mathcal{Z}_{\text {equi }}\left(X_{\alpha} \times_{T_{\alpha}}-/-, 0\right)_{\mathbb{Q}}$ is the inverse image of the Zariski sheaf $\mathcal{Z}_{\text {equi }}\left(X_{\alpha} \times_{T_{\alpha}}-/-, 0\right)_{\mathbb{Q}}$ on $T_{\alpha}$.

Proof. Let $\mathcal{T}_{\alpha}$ be the category of Zariski-open subschemes of $T_{\alpha}$. Note that the family of inverse images

$$
\left\{f_{\alpha}^{-1}\left(U_{\alpha}\right) \mid U_{\alpha} \in \mathcal{T}_{\alpha}, f_{\alpha \beta}^{-1}\left(U_{\alpha}\right)=U_{\beta} \text { for } \beta \geqslant \alpha, \alpha \in I\right\}
$$

is an open basis of $X \times_{S} T$. We prove that the canonical morphism

$$
\varliminf_{\beta \geqslant \alpha} \mathcal{Z}_{\text {equi }}\left(X_{\beta} \times{ }_{S} U_{\beta} / U_{\beta}, 0\right)_{\mathbb{Q}} \rightarrow \mathcal{Z}_{\text {equi }}\left(X \times_{S} U / U, 0\right)_{\mathbb{Q}}
$$

is bijective. The injectivity is obvious. We prove its surjectivity. Let $[W] \in \mathcal{Z}_{\text {equi }}\left(X \times_{T}\right.$ $U / U, 0)_{\mathbb{Q}}$ be the cycle of an integral scheme $W \subset X \times_{T} U$. Since $W \rightarrow U$ is quasifinite, there exists an index $\gamma$ and a closed integral subscheme $W_{\gamma} \subset X_{\gamma} \times_{T_{\gamma}} U_{\gamma}$ such that $W=W_{\gamma} \times_{U_{\gamma}} U$ and each $W \times_{T_{\gamma}} T_{\gamma^{\prime}} \rightarrow U_{\gamma} \times_{T_{\gamma}} T_{\gamma^{\prime}}$ is quasi-finite for $\gamma^{\prime} \geqslant \gamma$ by [4, Theorem 8.10.5]. Since $W \rightarrow U$ and $U \rightarrow U_{\gamma}$ are dominant, $W_{\gamma} \rightarrow U_{\gamma}$ is dominant. Hence the cycle $\left[W_{\gamma}\right]$ is in $\mathcal{Z}_{\text {equi }}\left(X_{\gamma} / U_{\gamma}, 0\right)$. By [8, Chapter 2, Lemma 3.3.6], $f_{\gamma}\left[W_{\gamma}\right]$ is a formal linear combination of irreducible components of $W=W_{\gamma} \times_{U_{\gamma}} U$, and by [8, Chapter 2, Lemma 3.5.9] there exists a positive integer $m$ such that $f_{\gamma}^{*}\left[W_{\gamma}\right]=m[W]$. Thus $[W]=f_{\gamma}^{*}\left(m^{-1}\left[W_{\gamma}\right]\right)$.

\section{The proof of main theorem}

\subsection{The cycle class maps}

In this section, we assume that all schemes are noetherian and separated. Let $\mathcal{C P}(X)$ be the category of bounded complexes of big vector bundles on $X$. Let $\mathcal{F}$ be a family of closed subschemes of $X$ and $\mathcal{C P} \mathcal{P}^{\mathcal{F}}(X)$ the full subcategory of $\mathcal{C P}(X)$ consisting of complexes acyclic outside of $\bigcup_{W \in \mathcal{F}} W$. We make $\mathcal{C} \mathcal{P}^{\mathcal{F}}(X)$ into a Waldhausen category by cofibrations and weak equivalences to be degree-wise split monomorphisms and quasi-isomorphisms, respectively. (See [7] and [9].)

Assume further that $f: Y \rightarrow X$ is a morphism of schemes and $\mathcal{F}^{\prime}$ is a family of closed subschemes of $Y$. The functor $f^{*}$ takes $\mathcal{C P} \mathcal{F}^{\mathcal{F}}(X)$ to $\mathcal{C P}^{\mathcal{F}^{\prime}}(Y)$ provided that $f^{-1}(W) \subset \bigcup_{W^{\prime} \in \mathcal{F}^{\prime}} W^{\prime}$ for all $W \in \mathcal{F}$. Furthermore, for a composition $Z \stackrel{g}{\rightarrow} Y \stackrel{f}{\rightarrow} X$ of morphisms of $X$-schemes, one has $(g \circ f)^{*}=f^{*} \circ g^{*}$ if $f^{*}, g^{*}$ and $(g \circ f)^{*}$ are defined.

Let $S$ be a regular noetherian scheme. For any regular noetherian schemes $X$, $S_{\bullet} \mathcal{C P}^{\mathcal{Q}, S}(X)$ denotes the Waldhausen's $S$-construction (cf. [9]) of $\mathcal{C P}^{\mathcal{Q}, S}(X):=$ $\mathcal{C P}^{\mathcal{Q}_{X}\left(X \times_{\mathbb{Z}} S\right)}(X \times S)$ with the family of supports $\mathcal{Q}_{X}\left(X \times \times_{\mathbb{Z}} S\right)$ consisting of all closed subschemes quasi-finite over $X$. Further, $K_{n}^{\mathcal{Q}, \mathcal{S}}(X)$ denotes the $n$-th $K$-group of $\mathcal{C} \mathcal{P}^{\mathcal{Q}, S}(X)$.

For any abelian group $A, B \bullet(A)$ denotes the classifying space of $A$. For any small category $\mathcal{C}, N_{\bullet}(\mathcal{C})$ denotes the nerve of $\mathcal{C}$. If $S=\mathbb{A}^{r}$, we define a map $\mathrm{cl}_{0}^{r}: B_{\bullet}\left(K_{0}^{\mathcal{Q}, \mathbb{A}^{r}}(X)\right) \rightarrow B_{\bullet}\left(\mathcal{Z}_{\text {equi }}\left(X \times \mathbb{A}^{r} / X, 0\right)\right)$ of simplicial sets by the formula

$$
\operatorname{cl}_{0}^{r}(\mathcal{F})=\sum_{W \subset X \times_{k} \mathbb{A}^{r}} \operatorname{length}\left(\mathcal{F}_{W}\right)[W],
$$


where the sum is over all closed integral subschemes $W$ of $X \times \mathbb{A}^{r}$ which are quasifinite and dominant over a component of $X$. We consider the composition

$$
\mathrm{cl}^{r}: N_{\bullet} \mathbf{w} S_{\bullet} \mathcal{C} \mathcal{P}^{\mathcal{Q}, \mathbb{A}^{r}}(X) \rightarrow B_{\bullet}\left(K_{0}^{\mathcal{Q}, \mathbb{A}^{r}}(X)\right) \stackrel{B_{\bullet}\left(\mathrm{cl}_{0}^{r}\right)}{\longrightarrow} B_{\bullet}\left(\mathcal{Z}_{\text {equi }}\left(X \times \mathbb{A}^{r} / X, 0\right)\right),
$$

where $\mathbf{w} S \cdot \mathcal{C P}^{\mathcal{Q}, \mathbb{A}^{r}}(X)$ is the subcategory of weak-equivalences in $S \cdot C \mathcal{P}^{\mathcal{Q}, \mathbb{A}^{r}}(X)$, and $\mathbf{w} S . C \mathcal{P}^{\mathcal{Q}, \mathbb{A}^{r}}(X) \rightarrow\left(K_{0}^{\mathcal{Q}, \mathbb{A}^{r}}(X)\right)^{n}$ is the canonical map of bisimplicial sets. (See $[7$, Section 1].)

For any morphism $f: Y \rightarrow X$ of regular noetherian schemes, $f^{*}: K_{0}^{\mathcal{Q}, \mathbb{A}_{\mathbb{Z}}^{r}}(X) \rightarrow$ $K_{0}^{\mathcal{Q}, \mathbb{A}_{\mathbb{Z}}^{r}}(Y)$ coincides with the map $\mathcal{F} \mapsto \sum_{i \geqslant 0}(-1)^{i} \mathbb{L}_{i} f^{*}(\mathcal{F})$, where each $\mathbb{L}_{i} f^{*}$ is the $i$-th left derived functor of the inverse image $f^{*}$. Using [8, Theorem 3.3.1 and Lemma 3.5.9], we have that the map $B .\left(\mathrm{cl}_{0}^{r}\right)$ is functorial for any morphism of regular noetherian schemes by the direct calculation. Hence $\mathrm{cl}^{r}$ is functorial for any regular noetherian schemes. In particular, $\mathrm{cl}^{r}$ commutes with all coface maps and codegeneracy maps of the regular noetherian cosimplicial scheme $X \times \Delta^{\bullet}$. Thus we obtain the map

$$
\begin{aligned}
\mathrm{cl}^{r}: N_{\bullet} \mathrm{w} S_{\bullet} \mathcal{C} \mathcal{P}^{\mathcal{Q}, \mathbb{A}^{r}}\left(X \times \Delta^{\bullet}\right) \rightarrow B_{\bullet}\left(K_{0}^{\mathcal{Q}, \mathbb{A}^{r}}\right. & \left.\left(X \times \Delta^{\bullet}\right)\right) \\
& \rightarrow B \bullet\left(\mathcal{Z}_{\text {equi }}\left(X \times \Delta^{\bullet} \times \mathbb{A}^{r} / X \times \Delta^{\bullet}, 0\right)\right)
\end{aligned}
$$

called the cycle-class map. Here $B_{\bullet}\left(A_{\bullet}\right)$ is the classifying space of a simplicial abelian group $A_{\bullet}$, and $B_{\bullet}\left(A_{\bullet}\right)$ is a bisimplicial set.

\subsection{Friedlander-Suslin's spectral sequence}

In this section, we consider the case where $X$ is smooth over a field $F$. Let $K_{n}^{\mathcal{Q}, \mathbb{A}^{r}}\left(X \times \Delta^{\bullet}\right)$ denote the $n+1$-th homotopy group of the diagonal of a 3 -fold simplicial set $N_{\bullet} \mathbf{w} S_{\bullet} \mathcal{C P}^{\mathcal{Q}, \mathbb{A}^{r}}\left(X \times \Delta^{\bullet}\right)$. In the case that $X$ is a smooth scheme over a field, Friedlander-Suslin [2] proved that there exists a strongly convergent spectral sequence:

$$
E_{2}^{p q}=\mathrm{CH}_{\text {Zar }}^{-q}(X,-p-q) \Longrightarrow K_{-p-q}(X)
$$

by an exact couple $\left(D_{2}^{p, q}, E_{2}^{p, q}, i, j, k\right)$ defined by the following:

$$
D_{2}^{p, q}=K_{-p-q}^{\mathcal{Q}, \mathbb{A}^{-q+1}}\left(X \times \Delta^{\bullet}\right), \quad E_{2}^{p, q}=\mathrm{CH}_{\mathrm{Zar}}^{-q}(X,-p-q),
$$

where $j$ is the cycle-class map. (See [2, Section 13].) We have that Friedlander-Suslin's spectral sequence admits Adams operations:

Proposition 3.1 (cf. [3, Theorem 7]). Let $X$ be a smooth scheme over a field F. Then the spectral sequence

$$
E_{2}^{p q}=\mathrm{CH}_{\text {Zar }}^{-q}(X,-p-q) \Longrightarrow K_{-p-q}(X)
$$

admits Adams operations $\Psi^{k}$ with the following properties:

(1) The $\Psi^{k}$ are natural in $\operatorname{Sm}_{F}$.

(2) The $\Psi^{k}: K_{*}^{\mathcal{Q}, \mathbb{A}^{q}}\left(X \times \Delta^{\bullet}\right) \rightarrow K_{*}^{\mathcal{Q}, \mathbb{A}^{q}}\left(X \times \Delta^{\bullet}\right)$ are compatible with the Adams operations $\Psi^{k}$ on $K_{*}(X)=K_{*}^{\mathcal{Q}, \mathbb{A}^{0}}(X)$.

(3) On the $E_{2}$-term $\mathrm{CH}_{\mathrm{Zar}}^{-q}(X,-p-q), \Psi^{k}$ acts by multiplication by $k^{-q}$.

Proof. The proof is similar to [3, Theorem 7]. 
Corollary 3.2. Let $X$ be a smooth scheme over a field $F$. The cycle-class map $\mathrm{cl}^{r}: K_{n}^{\mathcal{Q}, \mathbb{A}^{r}}\left(X \times \Delta^{\bullet}\right) \rightarrow \mathrm{CH}_{\mathrm{Zar}}^{r}(X, n)_{\mathbb{Q}}$ induces an isomorphism

$$
\mathrm{cl}^{(r)}: K_{n}(X)_{\mathbb{Q}}^{(r)} \rightarrow \mathrm{CH}_{\text {Zar }}^{r}(X, n)_{\mathbb{Q}}
$$

for any $n, r \geqslant 0$.

\subsection{The proof of Theorem 1.1}

By Popescu's result [6, Corollary 2.7], there exist a prime field $F$ and a directed inductive system $\left(R_{\alpha}, \psi_{\beta \alpha}\right)$ of smooth $F$-algebras of $R$ such that its inductive limit is $R$. Since each $\psi_{\beta \alpha}^{\sharp}$ : Spec $R_{\beta} \rightarrow \operatorname{Spec} R_{\alpha}$ is affine, we have that $\lim _{\mathrm{CH}}^{r}\left(R_{\alpha}, n\right)_{\mathbb{Q}}=$ $\mathrm{CH}_{\text {Zar }}^{r}(R, n)_{\mathbb{Q}}$ follows from [5, Theorem 5.7] and Proposition 2.2. By the functoriality of cycle-class maps and Corollary 3.2, we obtain

$$
K_{n}(R)_{\mathbb{Q}}^{(r)}=\lim _{\longrightarrow} K_{n}\left(R_{\alpha}\right)_{\mathbb{Q}}^{(r)}=\lim _{\longrightarrow} \mathrm{CH}_{\mathrm{Zar}}^{r}\left(R_{\alpha}, n\right)_{\mathbb{Q}}=\mathrm{CH}_{\mathrm{Zar}}^{r}(R, n)_{\mathbb{Q}} .
$$

\section{References}

[1] S. Bloch, Algebraic cycles and higher K-theory, Adv. in Math. 61 (1986), no. 3, 267-304.

[2] E.M. Friedlander and A. Suslin, The spectral sequence relating algebraic K-theory to motivic cohomology, Ann. Sci. École Norm. Sup. (4) 35 (2002), no. 6, 773-875 (English, with English and French summaries).

[3] H. Gillet and C. Soulé, Filtrations on higher algebraic K-theory, Algebraic $K$-theory (Seattle, WA, 1997), Proc. Sympos. Pure Math., vol. 67, Amer. Math. Soc., Providence, RI, 1999, pp. 89148.

[4] A. Grothendieck, Éléments de géométrie algébrique. IV. Étude locale des schémas et des morphismes de schémas. $3^{\text {eme }}$ partie, Inst. Hautes Études Sci. Publ. Math. 28 (1966), 5-255.

[5] A. Dold and B. Eckmann (ed.), Théorie des topos et cohomologie étale des schémas. Tome 2, Lecture Notes in Mathematics 270, Springer-Verlag, New York, 1972 (French). Séminaire de Géométrie Algébrique du Bois-Marie 1963-1964 (SGA 4); Dirigé par M. Artin, A. Grothendieck et J.L. Verdier. Avec la collaboration de N. Bourbaki, P. Deligne et B. Saint-Donat.

[6] D. Popescu, General Néron desingularization and approximation., Nagoya Math. J. 104 (1986), no. Teor. Chisel, Algebra i Algebr. Geom., 85-115.

[7] R.W. Thomason and T. Trobaugh, Higher algebraic K-theory of schemes and of derived categories, The Grothendieck Festschrift, Vol. III, Progr. Math., vol. 88, Birkhäuser Boston, Boston, MA, 1990, pp. 247-435.

[8] V. Voevodsky, A. Suslin, and E.M. Friedlander, Cycles, transfers, and motivic homology theories, Annals of Mathematics Studies, vol. 143, Princeton University Press, Princeton, NJ, 2000.

[9] F. Waldhausen, Algebraic K-theory of spaces, Algebraic and geometric topology (New Brunswick, NJ, 1983), Lecture Notes in Math., vol. 1126, Springer-Verlag, New York, 1985, pp. 318-419.

Yuki Kato ykato.math@gmail.com

Mathematical Institute, Tohoku University, Aoba, Sendai 980-8578 Japan 